\title{
The role of the Fraunhofer lines in solar brightness variability
}

\author{
A. I. Shapiro ${ }^{1}$, S. K. Solanki ${ }^{1,2}$, N. A. Krivova ${ }^{1}$, R. V. Tagirov ${ }^{3,4}$, and W. K. Schmutz ${ }^{3}$ \\ ${ }^{1}$ Max-Planck-Institut für Sonnensystemforschung, Justus-von-Liebig-Weg 3, 37077 Göttingen, Germany \\ e-mail: shapiroa@mps.mpg.de \\ 2 School of Space Research, Kyung Hee University, Yongin, 446-701 Gyeonggi, Korea \\ 3 Physikalisch-Meteorologishes Observatorium Davos, World Radiation Centre, 7260 Davos Dorf, Switzerland \\ ${ }^{4}$ Institute of Astronomy, ETH Zentrum, 8092 Zurich, Switzerland
}

Received 7 May 2015 / Accepted 12 July 2015

\section{ABSTRACT}

\begin{abstract}
Context. The solar brightness varies on timescales from minutes to decades. A clear identification of the physical processes behind such variations is needed for developing and improving physics-based models of solar brightness variability and reconstructing solar brightness in the past. This is, in turn, important for better understanding the solar-terrestrial and solar-stellar connections.

Aims. We estimate the relative contributions of the continuum, molecular, and atomic lines to the solar brightness variations on different timescales.

Methods. Our approach is based on the assumption that variability of the solar brightness on timescales greater than a day is driven by the evolution of the solar surface magnetic field. We calculated the solar brightness variations employing the solar disc area coverage of magnetic features deduced from the $\mathrm{MDI} / \mathrm{SOHO}$ observations. The brightness contrasts of magnetic features relative to the quiet Sun were calculated with a non-LTE radiative transfer code as functions of disc position and wavelength. By consecutive elimination of molecular and atomic lines from the radiative transfer calculations, we assessed the role of these lines in producing solar brightness variability.

Results. We show that the variations in Fraunhofer lines define the amplitude of the solar brightness variability on timescales greater than a day and even the phase of the total solar irradiance variability over the 11-year cycle. We also demonstrate that molecular lines make substantial contribution to solar brightness variability on the 11-year activity cycle and centennial timescales. In particular, our model indicates that roughly a quarter of the total solar irradiance variability over the 11-year cycle originates in molecular lines. The maximum of the absolute spectral brightness variability on timescales greater than a day is associated with the CN violet system between 380 and $390 \mathrm{~nm}$.
\end{abstract}

Key words. Sun: activity - solar-terrestrial relations - Sun: magnetic fields - Sun: faculae, plages - sunspots - line: formation

\section{Introduction}

Regular spaceborne measurements of the total solar irradiance (TSI, which is the spectrally integrated solar radiative flux at one au from the Sun) started in 1978 with the launch of the NIMBUS 7 mission (Hoyt et al. 1992). They revealed that the TSI varies on multiple timescales (Willson et al. 1981; Fröhlich 2005). The most striking features of the TSI records are the $\sim 0.1 \%$ modulation over the course of the 11-year solar activity cycle and more irregular variations on the timescale of solar rotation with amplitudes of up to $0.3 \%$.

Measurements of the spectral solar irradiance (SSI, which is the solar radiative flux per unit wavelength at one au from the Sun) showed the variability on the same two timescales (see Floyd et al. 2003; Harder et al. 2009; Deland \& Cebula 2012, and references therein). They also indicated that the amplitude of the relative SSI variations strongly depends on the wavelength and generally increases towards short wavelengths. For example, the variability of the solar Ly- $\alpha$ line $(121.5 \mathrm{~nm})$ irradiance over the course of the 11-year cycle reaches $100 \%$ (Woods et al. 2000); i.e., it is three orders of magnitude higher than the TSI variability.

It is widely accepted now that the SSI variability on timescales of a day and longer is caused by solar surface magnetic fields (Domingo et al. 2009; Krivova et al. 2011a; Ermolli et al. 2013; Solanki et al. 2013; Yeo et al. 2014a), although several other physical mechanisms for SSI variability have been proposed (see e.g. Kuhn \& Stein 1996; Kuhn et al. 1998).

Magnetic fields emerge on the solar surface in the form of magnetic concentrations described well by flux tubes (see Solanki 1993; Solanki et al. 2006, for reviews). Large flux tubes usually form dark sunspots, while small flux tubes appear as bright features ensembles of which compose faculae and the network, while individual small flux tubes are seen as bright points when observed at high resolution. The effects from the dark and bright features on solar irradiance do not compensate each other and the imbalance depends on the solar disc coverage by magnetic features of different sizes and on the wavelength observed. The coverage changes with time, leading to the variability of solar irradiance on timescales from days to decades. In recent years a number of models of solar irradiance variability have been created that are to some extent based on such a concept (e.g. Fligge et al. 2000; Krivova et al. 2003; Lean et al. 2005; Shapiro et al. 2011b; Fontenla et al. 2011; Bolduc et al. 2014; Ball et al. 2014; Yeo et al. 2014b).

The interest in solar irradiance variability is not limited to the solar community. The terrestrial climate responds to the decadal variations in solar irradiance (see e.g. reviews by Haigh 2007; Gray et al. 2010; Ermolli et al. 2013; Solanki et al. 2013) and there is also evidence for a longer term influence of solar activity on climate (e.g. Bond et al. 2001). Studies of solar irradiance 
are also of high importance for stellar astronomers, who have been comparing it with the variability of other lower main sequence stars (Radick et al. 1998; Lockwood et al. 2007; Hall et al. 2009). The interest in solar-stellar comparisons has recently been rekindled (see e.g. McQuillan et al. 2012; Basri et al. 2013) by the unprecedented precision of broadband stellar photometry achieved with the launch of the Kepler (Borucki et al. 2010) and Corot (Bordé et al. 2003; Baglin et al. 2006) space missions and the anticipation of the upcoming PLATO mission (Rauer et al. 2014).

Changes in the solar spectrum can be decomposed into the variations in the continuum and in spectral lines. The spectral lines are present all over the solar spectrum and they are responsible for its extremely rich and sophisticated structure. In the EUV (10-100 nm) and far UV (100-200 nm) below $170 \mathrm{~nm}$ the solar spectrum contains strong emission lines, while longward of $170 \mathrm{~nm}$ its profile is determined by many millions of atomic and molecular absorption lines, also called Fraunhofer lines. It is hardly possible to find a spectral interval without Fraunhofer lines in the visible and near-infrared parts of the solar spectrum. In the UV the immense number of spectrally unresolved lines form the UV line haze which completely blocks the continuum photons (e.g. Collet et al. 2005; Short \& Hauschildt 2005).

The variations in the Fraunhofer lines in the full-disc solar brightness spectrum over the course of the activity cycle were first revealed in the pioneering studies by Livingston \& Holweger (1982) who analysed the Kitt Peak spectral records for seven Fraunhofer lines and found a decrease of equivalent width ranging from no variation ( $\mathrm{Si}$ I 10827.1 $\AA$ line) to $2.3 \%$ (C I $5380.3 \AA$ line) from 1976 to 1980 . Livingston \& Holweger (1982) suggested that this decrease may be attributed to a change in the photospheric temperature gradient (see also Holweger et al. 1983). Later Mitchell \& Livingston (1991) employed Fourier transform spectrometer (FTS) observations at the McMath Telescope to show that the absorption lines between 500 to $560 \mathrm{~nm}$ exhibited a $1.4 \%$ decrease in amplitude and $0.8 \%$ decrease in equivalent width, at maximum compared with minimum of cycle 21. Interestingly, Livingston et al. (2007) could not confirm this finding in the next solar cycle for the unblended strong Fe I and the Na I D lines. This is, probably, a good indication of how challenging such observations are (see also discussion in Penza et al. 2006). Instead, Livingston et al. (2007) reported cyclic variation in the $\mathrm{Mn}$ I $539.4 \mathrm{~nm}$ line and the CN $388.3 \mathrm{~nm}$ bandhead.

Efforts have also been made to estimate the effect of the Fraunhofer lines on the SSI variability with models. For example, Unruh et al. (1999) used spectra computed using the probability distribution functions and an estimate of spot and facular disc area coverages during the solar maximum to show that spectral lines are the major contributor to the TSI variations in the 11-year activity cycle timescale rather than the continuum. In this study we follow up on the idea of Unruh et al. (1999) and employ the SATIRE (Spectral And Total Irradiance Reconstruction, see Fligge et al. 2000; Krivova et al. 2003) model to assess the relative role of continuum and spectral lines contributions to the SSI variability. The availability of stateof-the-art measurements of solar disc area coverage of magnetic features and radiative transfer calculations of their spectra, that now account for the effects of non Local Thermodynamical Equilibrium (non-LTE), allows us to define the contribution of spectral lines to the irradiance variability over the entire solar spectrum (including the UV) and on different timescales of variability.
In Sect. 2 we describe our model. In Sect. 3 we show how the Fraunhofer lines affect the brightness contrasts between the different magnetic features and quiet regions on the solar surface. In Sect. 4 we define the spectral profiles of the irradiance variability on the solar rotational, 11-year activity cycle, and centennial timescales. In Sect. 5 we show how these three profiles look if we first exclude molecular lines from our calculations and then all Fraunhofer lines. The main results are summarised in Sect. 6.

\section{The model}

We base our calculations on the SATIRE model. Its branch SATIRE-S (with "S" standing for the satellite era, see Krivova et al. 2011b) has been updated and refined over the recent years (Ball et al. 2012, 2014; Yeo et al. 2014b) and it currently replicates over $92 \%$ of the observed TSI variability over the entire period of spaceborne observations.

In SATIRE magnetic features observed on the solar disc are divided into three classes: sunspot umbrae, sunspot penumbrae, and the combined faculae and the network. The part of the solar disc not covered by these magnetic features is attributed to the quiet Sun. The brightness of each component (i.e. one of the three magnetic feature classes or the quiet Sun) is assumed to be time-invariant, but depends on the wavelength and position on the visible solar disc. The solar irradiance is calculated by weighting the spectra of the individual components with corresponding disc area coverages, i.e.:

$$
S(t, \lambda)=\sum_{k} \iint_{\text {solar disc }} I_{k}(\lambda, \boldsymbol{r}) \alpha_{k}(t, \boldsymbol{r}) \mathrm{d} \Omega,
$$

where the summation is done over the three SATIRE classes of magnetic features and the quiet Sun. For each of the components the integration is performed over the visible solar disc. Here $I_{k}(\lambda, r)$ is the emergent intensity from the component $k$ at the wavelength $\lambda$ along the direction $\boldsymbol{r}$. The functions $\alpha_{k}(t, \boldsymbol{r})$ are the fractional coverages of the solar disc by the component $k$ along the direction $\boldsymbol{r}$, so that $\alpha_{k}(t, \boldsymbol{r}) \mathrm{d} \Omega$ gives the elementary solid angle covered by the component $k$.

The two main building blocks of our model are the spectra $I_{k}(\lambda, r)$ and the fractional coverages $\alpha_{k}(t, r)$ of the quiet Sun and magnetic features. We utilise the fractional coverages deduced by Ball et al. (2012) from the full-disc continuum images and magnetograms obtained by the Michelson Doppler Imager onboard the Solar and Heliospheric Observatory (SOHO/MDI; Scherrer et al. 1995). Ball et al. (2012) successfully used these fractional coverages to reproduce $96 \%$ of TSI variations during cycle 23 (represented by the PMOD composite by Fröhlich 2006). Following Ball et al. $(2011,2012)$ we replace the integration in Eq. (1) with the summation over magnetogram pixels. Then the $\alpha_{k}(t, \boldsymbol{r})$ is substituted by $\alpha_{k i j}(t)$ which represents the coverage of the pixel with abscissa $i$ and ordinate $j$ by the component $k$. The $\alpha_{k i j}(t)$ values lie between 0 and 1 for the quiet Sun and faculae, and either 1 or 0 for umbra and penumbra.

The brightness spectra of magnetic features and the quiet Sun $I_{k}(\lambda, \boldsymbol{r})$ are calculated with Non local thermodynamic Equilibrium Spectral SYnthesis code (NESSY, Tagirov, priv. comm.), which is a further development of the COde for Solar Irradiance (COSI, Shapiro et al. 2010). This is in contrast to previous SATIRE publications, which have been based on spectra calculated with the ATLAS9 code by Kurucz (1992) and Castelli \& Kurucz (1994). NESSY simultaneously solves the statistical equilibrium equations for the elements from hydrogen to zinc, 
taking the coupling between level populations of different elements via the electron concentration and radiation field into account. The employment of NESSY allows us to recalculate the $I_{k}(\lambda, r)$ spectra over the entire frequency domain (in particular, including the UV, see Sect. 4) with and without molecular/Fraunhofer lines.

We adopt the 1D temperature and density structures of the quiet Sun, faculae, sunspot umbra, and sunspot penumbra from Models C, P, S by Fontenla et al. (1999), and Model R by Fontenla et al. (2006), respectively. The spectra emergent from magnetic features are calculated neglecting the Zeeman splitting. The populations of atomic and molecular levels, as well as the electron concentration are self-consistently calculated with NESSY. The atomic linelist is compiled from the "long" linelist provided by Kurucz (priv. comm.) and the Vienna Atomic Line Database (VALD, Kupka et al. 1999, 2000). The molecular linelist is complied from the list by Kurucz (1993) and the Solar Radiation Physical Modeling database (SRPM, see e.g. Fontenla et al. 2011). The strongest vibrational bands of the $\mathrm{CN}$ violet system and $\mathrm{CH} G$-band are calculated using the molecular constants by Krupp (1974), Knowles et al. (1988), Wallace et al. (1999) (see also Shapiro et al. 2010, 2011a, and references therein for more details).

The full disk brightness spectrum of the quiet Sun calculated with such a setup is in good agreement with various spaceborne measurements of the solar spectrum around activity minimum conditions (see Shapiro et al. 2010; Thuillier et al. 2011, 2014, 2015, for a detailed intercomparison). The centre-to-limb variations in the quiet Sun brightness agree well with measurements in the UV (Shapiro et al. 2013) and visible (Tagirov, priv. comm.) spectral domains. For the experiments presented below we use NESSY spectra calculated with the full molecular and atomic linelist, spectra produced excluding molecular lines, and finally spectra calculated excluding all Fraunhofer lines, i.e. when the total opacity is given only by the free-free and bound-free processes.

\section{Effect of the Fraunhofer lines on contrasts of solar magnetic features}

In the upper left panel of Fig. 1 we plot the spectral fluxes at $1 \mathrm{AU}$ (hereafter referred as brightness spectra or just brightness) emergent from the quiet Sun, faculae, spot umbrae and penumbrae calculated as discussed in Sect. 2. For illustration purposes the brightness of each type of magnetic feature is plotted assuming that it fully covers the visible solar disc.

With the exception of the umbral brightness, the spectral profiles of the brightness of different magnetic components are strongly affected by numerous absorption features. The umbral brightness is significantly lower than brightnesses of all other magnetic components considered in this study and the spectral lines have only marginal contribution to the umbral brightness contrast with respect to the quiet Sun. Hence the effect of spectral lines on the umbral spectrum will not be discussed in detail, though it will be taken into account when computing irradiance variability.

In the lower left panel of Fig. 1 we present facular and penumbral brightness contrasts with respect to the quiet Sun. They are calculated by subtracting the brightness of the quiet Sun from the facular and umbral brightness. The overall profiles of the contrasts are to some extent reminiscent of the solar spectrum but skewed towards the UV because at short wavelengths (i.e. when the Planck function can be approximated by the Wien approximation) the derivative of the Planck function with respect to the temperature (which defines the contrast) is proportional to the Planck function divided by the wavelength.

To illustrate the contribution of spectral lines to the facular and penumbral brightness contrasts we also show brightness spectra and contrasts calculated by putting the opacity of molecular lines to zero (middle panels of Fig. 1) as well as putting opacity in all Fraunhofer lines to zero (i.e. producing purely continuum spectra and contrasts, see right panels of Fig. 1). We discuss these plots in Sects. 3.1 and 3.2, respectively.

\subsection{Molecular lines}

The middle panels of Fig. 1 illustrate that the elimination of molecular lines from the radiative transfer calculations has a strong effect on the brightness spectra and the contrasts in the $300-450 \mathrm{~nm}$ spectral domain. The strongest peak in the facular contrast is linked to the $C N$ violet system $\left(B^{2} \Sigma-X^{2} \Sigma\right.$ transitions) between $380 \mathrm{~nm}$ and $390 \mathrm{~nm}$, while the second strongest peak results from the $\mathrm{CH} G$-band $\left(A^{2} \Delta-X^{2} \Pi\right.$ transitions $)$ around $430 \mathrm{~nm}$. These molecular systems, being also prominent features of the solar spectrum, almost double the facular contrast at the corresponding wavelengths. The brightness contrasts and spectra between 300 and $350 \mathrm{~nm}$ are also noticeably affected by the $\mathrm{CN}, \mathrm{NH}$, and $\mathrm{OH}$ bands.

The amplification of facular contrast by molecular lines is due to the strong sensitivity of molecular concentrations to temperature changes. Molecular concentrations are lower in faculae which are generally warmer than the quiet Sun surroundings. Consequently, the molecular lines in the facular spectrum are weaker than in the spectrum of the quiet Sun, which leads to the enhancement of the contrast. Such an interpretation is in line with Steiner et al. (2001), Sánchez Almeida et al. (2001). Similarly to this study, they employed 1D models of faculae and quiet Sun to explain the appearance of the $G$-band bright points, observed on $G$-band filtergrams, by the weakening of $\mathrm{CH}$ lines in hot flux tubes. Similar conclusion was later drawn by Schüssler et al. (2003) who employed more realistic 3D MHD simulations (see also Riethmüller et al. 2014, who studied the $\mathrm{CN}$ bright points).

The $\mathrm{CN}$ violet system and $\mathrm{CH} G$-band induce a comparable amplification of the facular brightness contrast even though the $\mathrm{CH} G$-band is more pronounced in the solar spectrum. This is partly caused by the relatively high dissociation energy of the $\mathrm{CN}$ molecule $(7.72 \mathrm{eV})$, which is larger than that of $\mathrm{CH}$ $(4.25 \mathrm{eV})$. As a consequence the $\mathrm{CN}$ concentration is more sensitive to temperature changes than the $\mathrm{CH}$ concentration (see e.g. the discussion of the chemical equilibrium calculations in Berdyugina et al. 2003).

The $\mathrm{CN}$ violet system and $\mathrm{CH} G$-band are less pronounced in the penumbral spectrum than in the spectrum of the quiet Sun, which is probably associated with a small gradient of photospheric temperature in the Fontenla et al. (2006) penumbral model (see their Fig. 6). As a result the penumbral brightness contrast drops in the $\mathrm{CH} G$-band and is only marginally affected by the $\mathrm{CN}$ violet system.

\subsection{Fraunhofer lines}

The right panels of Fig. 1 illustrate that the elimination of all Fraunhofer lines from the radiative transfer calculations has a profound effect on both the brightness spectra and the contrasts below about $600 \mathrm{~nm}$. The spectral lines are a dominant source of 

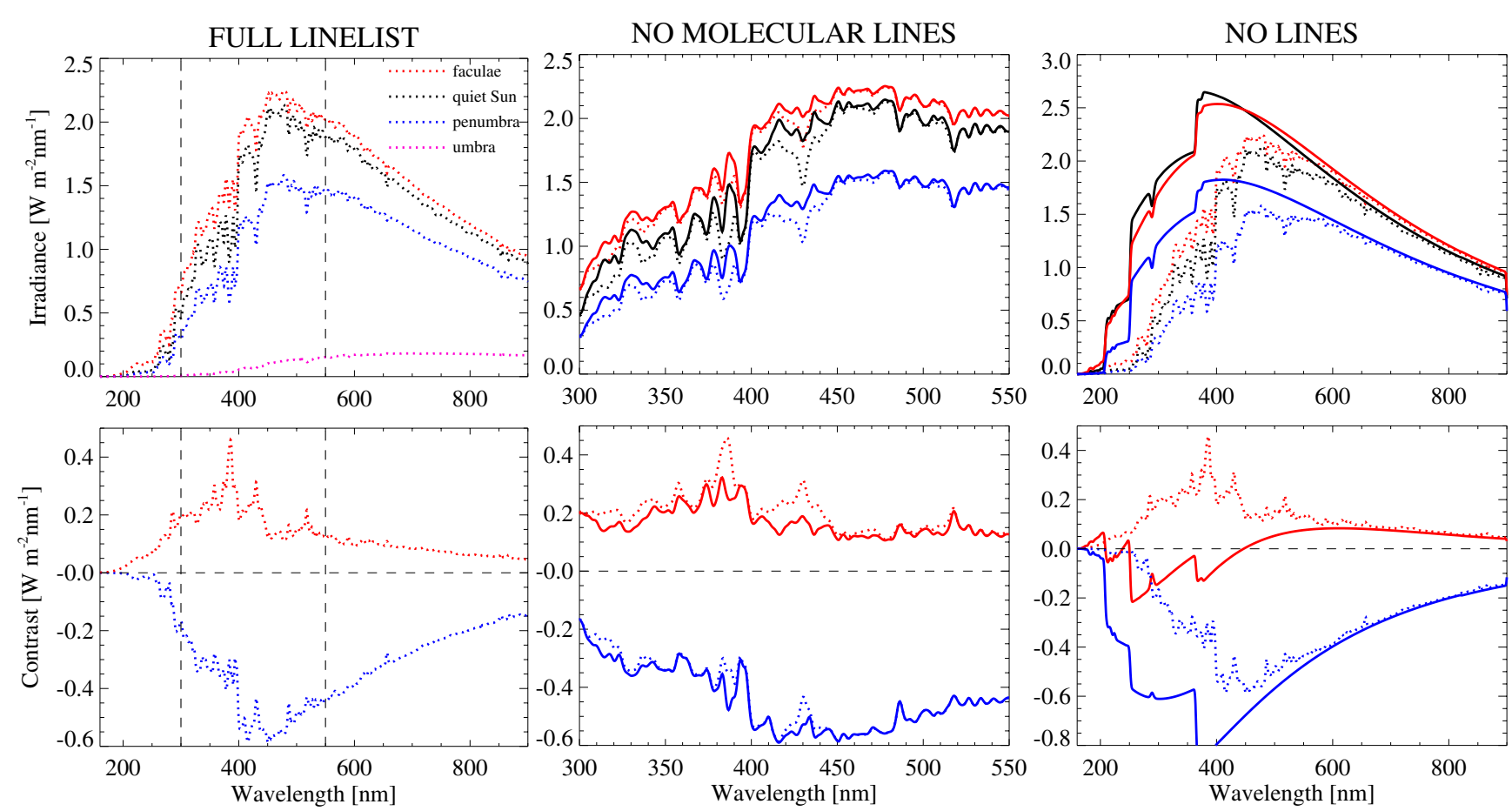

Fig. 1. Upper panels: brightness spectra of the quiet Sun (black curves), faculae (red curves), spot penumbra (blue curves) and umbra (magenta curve, only plotted in the upper left panel). Lower panels: facular (red curves) and penumbral (blue curves) brightness contrasts with respect to the quiet Sun. The dotted curves are calculated taking the spectral lines (full linelist) into account. The solid curves are calculated by putting the opacity in the molecular (middle panels) and in all spectral lines (right panels) to zero. To facilitate the comparison between different spectra and contrasts the dotted curves are plotted in all three panels. The two vertical dotted lines in the left panel constrain the spectral interval shown in the middle panels.

opacity in the UV and they significantly contribute to the total opacity in the visible spectral domain. By shifting the formation height of the radiation to higher and colder photospheric layers, the Fraunhofer lines significantly reduce the brightness of the quiet Sun, faculae, and penumbrae (see upper right panel of Fig. 1). The salient features of the continuum spectra are the Balmer, $\mathrm{Mg}$ I, and $\mathrm{Al} \mathrm{I}$ ionisation edges at $364.5 \mathrm{~nm}, 251.2 \mathrm{~nm}$, and $207.8 \mathrm{~nm}$, respectively. The feature around $290 \mathrm{~nm}$ is attributed by Haberreiter et al. (2003) to a resonance in the Mg I 3 photoionisation cross section. While the $\mathrm{Mg}$ I and $\mathrm{Al}$ I edges can still be recognised in the "real" (i.e. calculated with the full linelist, see dotted lines in Fig. 1) solar spectrum, the Balmer edge is completely hidden in the multitude of spectral lines.

The difference between the quiet sun and faculae continua is small only, the former slightly brighter in the middle and near UV (200-300 $\mathrm{nm}$ and 300-400 nm, respectively), the latter slightly brighter shortward of $200 \mathrm{~nm}$ and longward of $400 \mathrm{~nm}$. The penumbra continuum is well below the other two, as can be expected from its effective temperature that is about $250 \mathrm{~K}$ lower than that of the quiet sun.

The Fraunhofer lines substantially increase the facular contrast and decrease the absolute value of the penumbral contrast. This is linked to the temperature structure of the $1 \mathrm{D}$ atmospheric models (see Sect. 2): the temperature difference between the quiet Sun and faculae decreases, while the temperature difference between the quiet Sun and penumbra increases, towards deeper photospheric layers (see Fig. 6 from Fontenla et al. 2006), where the continuum intensity is formed. The effect of Fraunhofer lines on the brightness spectra and contrasts gets smaller in the infrared where the relative contribution of lines to the total opacity diminishes.

According to our calculations the facular contrast in the continuum becomes negative in the near and middle UV. The continuum irradiance at these wavelengths comes from the deepest photospheric layers (see also Ayres 1989). Indeed, at shorter wavelengths the photoionisation opacity from metals increases, while at longer wavelengths the opacity from negative hydrogen ions get higher (see Fig. 4.2 from Mihalas 1978). In deep photospheric layers faculae are cooler than the quiet Sun according to the 1D atmospheric structures employed here, which results in the inversion of the contrast. A similar inversion in the continuum contrast was recently obtained by Criscuoli \& Uitenbroek (2014) who based their calculations on 3D MHD simulations calculated by Fabbian et al. (2012) using STAGGER code (Galsgaard \& Nordlund 1996).

The negative full-disc facular continuum brightness contrasts in the UV obtained from our calculations are qualitatively consistent with the observations of negative continuum brightness contrasts of faculae about disc centre in the visible part of the solar spectrum (Yeo et al. 2013, and references therein) where the radiation also emanates from the deep photosphere. We note that the contribution functions (see e.g. Gray 1992, p. 151) for the full-disk UV and disc centre visible continuum radiation are comparable because the formation height of the continuum radiation decreases with cosine of the heliocentric angle but increases from the UV to visible. At the same time we are aware that while 1D models are designed to replicate many features of brightness spectra and contrasts (see e.g. Unruh et al. 1999; Fontenla et al. 2011) they miss the real physics of centre-to-limb variations in the facular contrast (Steiner 2005).

\section{Spectral irradiance variability on different timescales}

The most accurate and uninterrupted records of solar irradiance variability available so far are the TSI records (see e.g. the most 

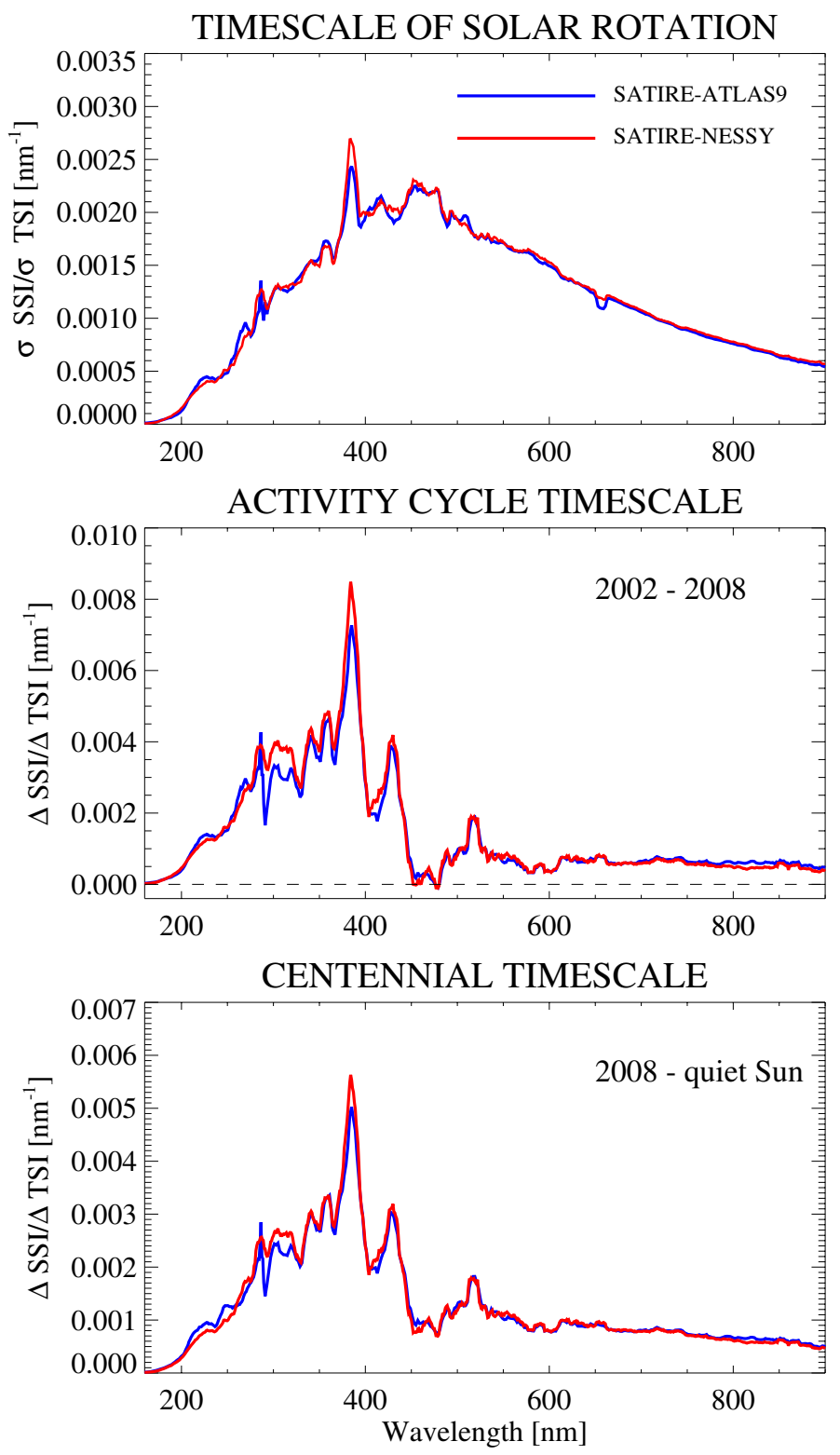

Fig. 2. Spectral profiles of the irradiance variability calculated on the solar rotational (upper panel), activity cycle (middle panel), and centennial (lower panel) timescales. Plotted are the values calculated employing "SATIRE-ATLAS9" SSI and TSI time series (blue curves) and values calculated employing the "SATIRE-NESSY" SSI and TSI time series (red curves). All spectral profiles are smoothed by applying a $10 \mathrm{~nm}$ running mean.

recent publications by Kopp et al. 2012; Fröhlich 2013; Schmutz et al. 2013; Kopp 2014, and references therein). Nevertheless more information than just TSI is needed for both climate simulations and the solar-stellar comparison. Climate simulations are very sensitive to the variability of the UV irradiance, which is the main driver in the top-down mechanism for the influence of the solar irradiance variability on climate (Haigh 1994; Kodera \& Kuroda 2002). Likewise, most stellar photometric records used for solar-stellar comparison are obtained with broad-band filters sensitive to the visible part of the spectrum (Lockwood et al. 2007; Hall et al. 2009; Basri et al. 2013). It is, therefore, important to know the spectral profiles of the solar irradiance variability on different timescales. In this section we explain how these spectral profiles are linked to the brightness contrast discussed in
Sect. 3. Then in Sect. 5 we show how these profiles are affected by the Fraunhofer lines.

We define the spectral profile of the irradiance variability on the timescale of solar rotation as:

$\frac{\sigma \mathrm{SSI}}{\sigma \mathrm{TSI}}(\lambda)=\frac{\operatorname{rms}\left(\operatorname{SSI}(\lambda, t)-\langle\operatorname{SSI}(\lambda, t)\rangle_{81}\right)}{\operatorname{rms}\left(\operatorname{TSI}(t)-\langle\operatorname{TSI}(t)\rangle_{81}\right)}$,

where "rms" stands for the root mean square. In our study both TSI and SSI time series are calculated with daily cadence so that the averaging in Eq. (2) represents the running mean over 81 days.

In the upper panel of Fig. 2 we plot $\sigma$ SSI $\sigma$ TSI values as functions of wavelength calculated with SATIRE-S TSI and SSI time series from Ball et al. (2012, 2014), hereafter "SATIRE-ATLAS9" time series, since they are based on the spectra of individual magnetic features and the quiet Sun calculated with the ATLAS9 code. These time series cover the period from February 19, 1999 till October 2, 2010 and are based on the fractional coverages of magnetic features deduced from the SOHO/MDI data. We also plot $\sigma \mathrm{SSI} / \sigma \mathrm{TSI}$ values calculated with the model described in Sect. 2, i.e. employing the same $\mathrm{SOHO} / \mathrm{MDI}$ fractional coverages of magnetic features as Ball et al. $(2012,2014)$ but convolving them with the NESSY spectra instead of the ATLAS9 spectra (see Sect. 2). These "SATIRENESSY" time series are calculated for the same period as the "SATIRE-ATLAS9" time series.

In the middle panel of Fig. 2 we plot "SATIRE-NESSY" and "SATIRE-ATLAS9" spectral profiles of the irradiance variability on the 11-year activity cycle timescale. They are defined as

$\frac{\Delta \mathrm{SSI}}{\Delta \mathrm{TSI}}(\lambda)=\frac{\langle\operatorname{SSI}(\lambda, t)\rangle_{2002}-\langle\operatorname{SSI}(\lambda, t)\rangle_{2008}}{\langle\operatorname{TSI}(t)\rangle_{2002}-\langle\operatorname{TSI}(t)\rangle_{2008}}$,

where annual averaging is performed over the calendar years of high (year 2002) and low (year 2008) solar activity.

The irradiance variability on a centennial timescale is often described as a secular change between solar minima conditions (see e.g. the discussion of TSI variability in Fröhlich 2009). The current solar irradiance records are too short and uncertain to unambiguously reveal and quantify secular changes. Consequently, the magnitude and even specific physical mechanisms responsible for the centennial SSI variability are heavily debated (see Solanki et al. 2013, and references therein). According to SATIRE most of the SSI changes between activity minima are caused by the varying contribution from the network component (Krivova et al. 2010). The contribution of this component does not drop to zero even at activity cycle minima and is responsible for the secular trend in solar irradiance, e.g. for the change in irradiance between the 2008 activity minimum and the Maunder minimum (however, see also Schrijver et al. 2011).

Along these lines we define the spectral profile of the irradiance variability on the centennial timescale as

$\frac{\Delta \mathrm{SSI}}{\Delta \mathrm{TSI}}(\lambda)=\frac{\langle\operatorname{SSI}(\lambda, t)\rangle_{2008}-\operatorname{SSI}_{\text {quiet }}(\lambda)}{\langle\operatorname{TSI}(t)\rangle_{2008}-\operatorname{TSI}_{\text {quiet }}}$,

where $\operatorname{SSI}_{\text {quiet }}(\lambda)$ and $\operatorname{TSI}_{\text {quiet }}$ are spectral and total solar irradiance, respectively, as they would be measured if the visible part of the solar disc did not contain any active features (i.e. was completely covered by the quiet Sun). We emphasise that our definition of the centennial variability refers to the irradiance changes between solar activity minima rather than averaged over the activity cycle as often done in the literature. The spectral profiles on the centennial timescale calculated with the "SATIRENESSY" and "SATIRE-ATLAS9" time series are plotted in the lowest panel of Fig. 2. 
In addition to strong concentrated magnetic fields, considered in SATIRE, there is also weak turbulent magnetic field on the solar surface (see reviews by de Wijn et al. 2009; Stenflo 2013). Its effect on the rotational and 11-year irradiance variability is believed to be small (cf. Solanki et al. 2013) but it is presently unclear whether this field can contribute to the irradiance variability on the centennial timescale (see e.g. discussion in Judge et al. 2012, and references therein). The ambiguity associated with this effect is an additional source of uncertainty in our estimate of the contributions of molecular and atomic lines to irradiance variability on the centennial timescale.

We note that the ATLAS9 code is based on the LTE assumption, which is not applicable in the UV. As a consequence Ball et al. (2014) corrected the SSI values between 115 and $270 \mathrm{~nm}$ based on the empirical method by Krivova et al. (2006). In contrast to ATLAS9, NESSY takes non-LTE effects into account and thus no additional correction of the "SATIRE-NESSY" time series is needed. Despite this difference in methods, the spectral profiles calculated with both time series are very close to each other over the entire spectral range shown in Fig. 2, also including the UV. We construe this as an independent support to both the Krivova et al. (2006) method and the reliability of the "SATIRE-NESSY" time series.

The "SATIRE-ATLAS9" SSI and TSI time series have been demonstrated to be consistent with observations from multiple sources (see Ball et al. 2014; Yeo et al. 2014b, and references therein). Since the differences between "SATIRE-NESSY" and "SATIRE-ATLAS9" spectral profiles are not essential for the purposes of this study we refrain from comparing the "SATIRENESSY" time series to observational data here, which is beyond the scope of this paper.

Figure 2 indicates that the spectral profile of the irradiance variability depends significantly on the considered timescale. The SSI variability on the 11-year activity cycle timescale (middle panel of Fig. 2) is brought about by the competition between bright faculae and network on the one hand and dark sunspots on the other hand. The 11-year TSI variability as well as the 11-year variability in the UV and visible are faculae-dominated and are in phase with the solar cycle (however, see also Harder et al. 2009), so that the $\Delta \mathrm{SSI} / \Delta \mathrm{TSI}$ values plotted in the middle panel of Fig. 2 are positive with the exception of a minor feature around $470 \mathrm{~nm}$. The facular contribution decreases with wavelengths faster than the spot contribution (in particular, the umbral component, see left upper panel of Fig. 1) so that starting from $600 \mathrm{~nm}$ both components almost cancel each other (while starting from 1-2 $\mu \mathrm{m}$ spots overweight the faculae and SSI varies out-of-phase with solar cycle, see e.g. Fig. 7 from Ermolli et al. 2013). As a result the dominant part of the irradiance variability over the 11-year activity cycle originates in the $250-450 \mathrm{~nm}$ spectral domain, which is especially affected by the molecular lines (see middle panels of Fig. 1).

The SSI variability on the timescale of solar rotation is mainly caused by transits of magnetic features across the visible solar disc as the Sun rotates. The spot and facular contributions to the irradiance rarely cancel each other at any specific moment of time. Hence, in contrast to the case of the 11-year variability, the amplitude of the irradiance variability on the rotational timescale does not drop in the visible part of the spectrum and is still significant in the red and IR spectral domains (where it is mainly associated with umbral contributions). Consequently the role of the wavelengths affected by the molecular lines is significantly smaller than in the case of the 11-year variability.

In our model the spectral profile of the irradiance variability on the centennial timescale is directly given by the spectral
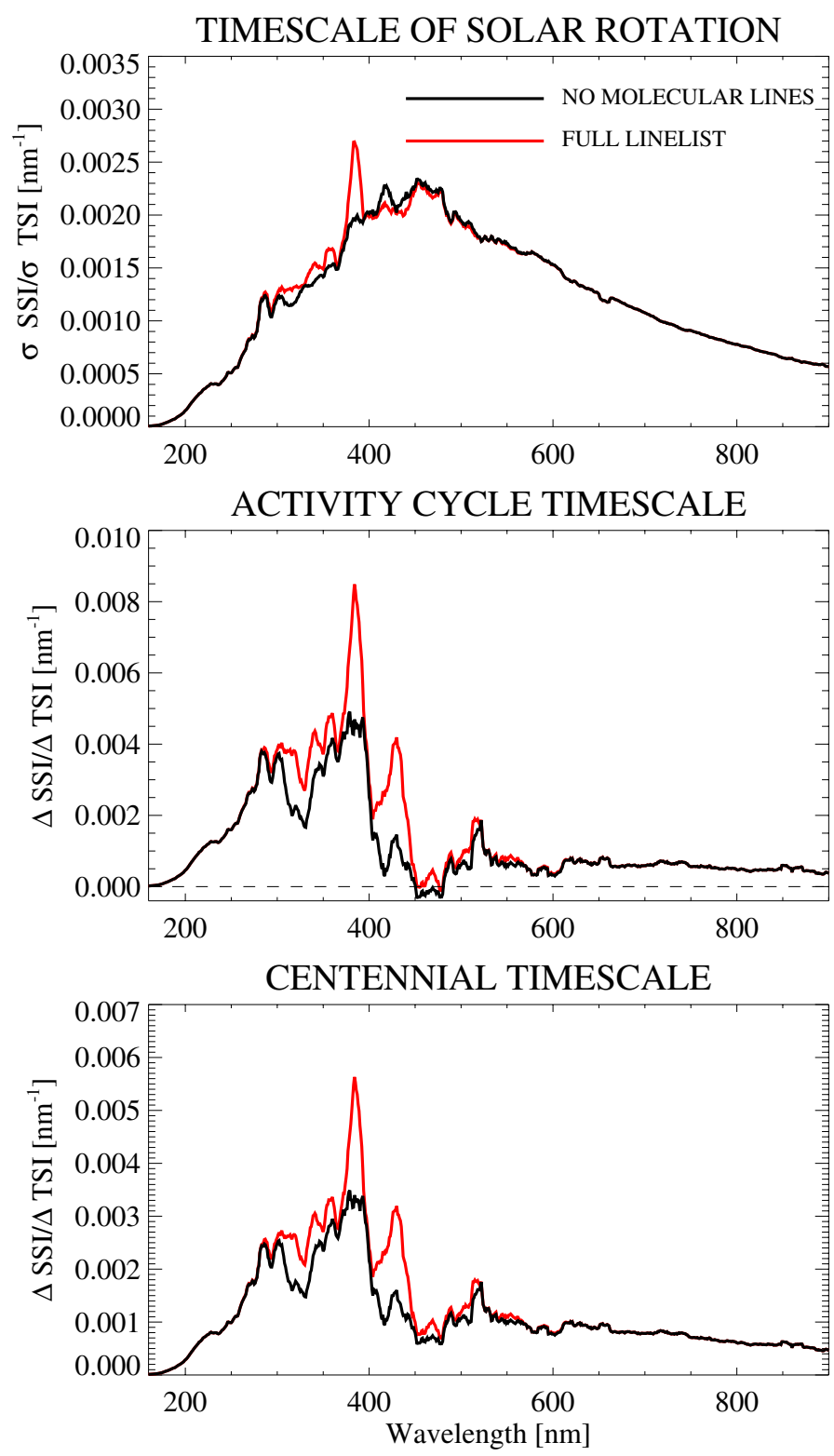

Fig. 3. Spectral profiles of the irradiance variability calculated on the solar rotational (upper panel), 11-year activity cycle (middle panel), and centennial (lower panel) timescales. Plotted are the values calculated with "SATIRE-NESSY" model taking the full linelist into account (red curves) as well as putting the opacity in molecular lines to zero (black curves).

profile of the facular brightness contrast (because the same atmospheric model is used for faculae and network, see Sect. 2). In the infrared the centennial SSI variability is larger than the 11-year SSI variability (since there is no competing effect from spots) but is substantially smaller than the variability on the timescale of solar rotation.

\section{Effect of the Fraunhofer lines on spectral irradiance variability}

Despite the difference in overall shape, all three spectral profiles of the irradiance variability, introduced in Sect. 4, have similar spectral features which are mostly attributed to different molecular bands and strong atomic lines. To pinpoint the contributions 
of variations in molecular and atomic lines to these profiles we calculated intensity spectra $I_{k}(\lambda, r)$ by first putting the opacity in molecular lines to zero and then the opacity in all Fraunhofer lines to zero. For each of the computations Eq. (1) was then used to produce "SATIRE-NESSY no molecules" and "SATIRE-

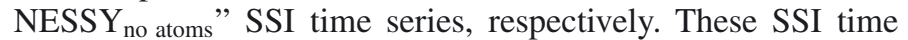
series were then substituted into Eqs. (2)-(4) to recalculate the spectral profiles of the irradiance variability as they would be measured without contributions from molecular/Fraunhofer lines. To maintain a consistent normalisation of the spectral profiles, the TSI time series used in Eqs. (2)-(4) was calculated taking the molecular and atomic lines into account, i.e. is always the same.

\subsection{Molecular lines}

In Fig. 3 we present a comparison of the spectral profiles of the SSI variability calculated employing the "SATIRENESSY" (produced with the full linelist) and "SATIRENESSY no molecules" SSI time series. In line with the discussion in Sect. 3.1, molecular lines mainly affect the near UV, violet, and blue spectral domains. They almost double the SSI variability on the 11-year and centennial timescales between 300 and $450 \mathrm{~nm}$. As mentioned in Sect. 4 this spectral domain also happens to be the major contributor to the TSI variability on the 11-year timescale. By integrating the spectral profiles of the centennial and 11-year variability over the wavelengths one can estimate the contribution of the molecular lines to the TSI variability. Our calculations indicate that $23 \%$ of the TSI variability on the 11 -year timescale is attributed to molecular lines. On the centennial timescale (bottom panel of Fig. 3) the relative contribution of the $300-450 \mathrm{~nm}$ spectral domain to the TSI variability is smaller than in the case of the 11-year variability. Consequently the contribution of molecular lines to the TSI variability on the centennial timescale is also slightly lower, being equal to $15 \%$.

In contrast to the 11-year and centennial variabilities, molecular lines do not play an essential role in the variability on the timescale of solar rotation. This is mainly because of the spot contribution which keeps the rotational variability high even in the absence of molecular lines.

Molecular lines are also responsible for the strongest peak in the SSI variability on all three timescales considered in this study, namely the $380-390 \mathrm{~nm}$ peak which is linked to the CN violet system. The second strongest peak in the SSI variability on the 11-year and centennial timescales is associated with the $\mathrm{CH}$ $G$-band.

\subsection{Fraunhofer lines}

In Fig. 4 we compare the spectral profiles calculated employing the "SATIRE-NESSY" and "SATIRE-NESSY no atoms" SSI time series. The elimination of the Fraunhofer lines has a profound effect on all three spectral profiles up to $600 \mathrm{~nm}$. The ionisation edges clearly visible in the brightness spectra and contrasts in the right panels of Fig. 1 are also prominent features of spectral profiles of continuum variability.

Notably our calculations indicate that the continuum SSI variability in the UV and visible is in antiphase with solar activity on the 11-year and centennial timescales. This is consistent with Unruh et al. (1999), who also reported an antiphase continuum variability on the 11-year timescale for the $300-500 \mathrm{~nm}$ spectral domain (cf. also Criscuoli \& Uitenbroek 2014).
Furthermore, since the 11-year and long-term TSI variability calculated without accounting for spectral lines is out-of-phase with the solar cycle, the contributions of Fraunhofer lines to the TSI variability on these timescales is larger than $100 \%$, being $250 \%$ and $140 \%$ for the 11-year and long-term timescales, respectively. By the same token the increase of the TSI at maximum of the activity cycle compared with its minimum is directly attributed to the variability in spectral lines. We note, however, that the exact behaviour of the continuum variability strongly depends on the temperature structures of the quiet Sun and magnetic features in the deepest photospheric layers. These layers provide a very small contribution to the emergent line spectra, so that it is difficult to reliably constrain their temperature structures in 1D semi-empirical modelling, especially taking intro account the uncertainties in the measurements of the IR solar irradiance which emanates from the deep photospheric layers (Thuillier et al. 2014; Bolsée et al. 2014; Thuillier et al. 2015). Consequently our model does not allow us to unambiguously rule out an in-phase continuum variability. At the same time our calculations clearly indicate that the variations in spectral lines govern the TSI variability on all three considered timescales.

\section{Discussion and conclusions}

We have estimated the contribution of the Fraunhofer lines to the solar irradiance variability driven by the evolution of the surface magnetic field on the solar rotational, 11-year activity cycle, and centennial timescales. Our calculations indicate that the solar irradiance variability in the UV, violet, blue, and green spectral domains is fully controlled by the Fraunhofer lines. The highest peak in absolute SSI variability on all considered timescales is associated with the $\mathrm{CN}$ violet system between 380 and $390 \mathrm{~nm}$. Furthermore, the molecular lines strongly enhance the SSI variability on the 11-year and centennial timescales. For example, according to our model, almost a quarter of the TSI variability on the 11-year timescale originates in molecular lines.

Such a substantial contribution of molecular lines to the irradiance variability is due to the strong sensitivity of molecular concentrations to temperature changes and consequently an enhanced molecular depletion in hot facular regions compared to the colder surrounding regions of the quiet Sun. This is a well known effect, which leads to the appearance of bright points in molecular lines at the locations of magnetic flux concentrations (Steiner et al. 2001; Schüssler et al. 2003) and makes molecular lines a sensitive tool for mapping photospheric temperatures and magnetic fields (Berdyugina et al. 2005). However, to our knowledge, it is the first time that molecular lines are directly linked to the enhancement of the irradiance variability.

Some words of caution are needed here since our model is based on the NLTE calculations with static 1D atmospheric stratifications of the quiet Sun and magnetic features. Such calculations are still being actively developed and are getting highly sophisticated (see e.g. discussion in Rutten \& Uitenbroek 2012). At the same time they are deemed (e.g. Koesterke et al. 2008; Uitenbroek \& Criscuoli 2011) to be inherently unreliable for diagnostics of solar atmospheric properties (see however Vitas et al. 2009), unless differential techniques are used (Stenflo et al. 1998). The 1D models cannot directly account for the hot walls of flux tubes which to large extent determine the centre-tolimb variation in facular brightness (Steiner 2005). Furthermore, molecular concentrations depend on the temperature in a nonlinear way (see e.g. Berdyugina et al. 2003) so that their response to horizontal temperature fluctuations cannot be properly averaged out in 1D calculations. Also, for some molecules 

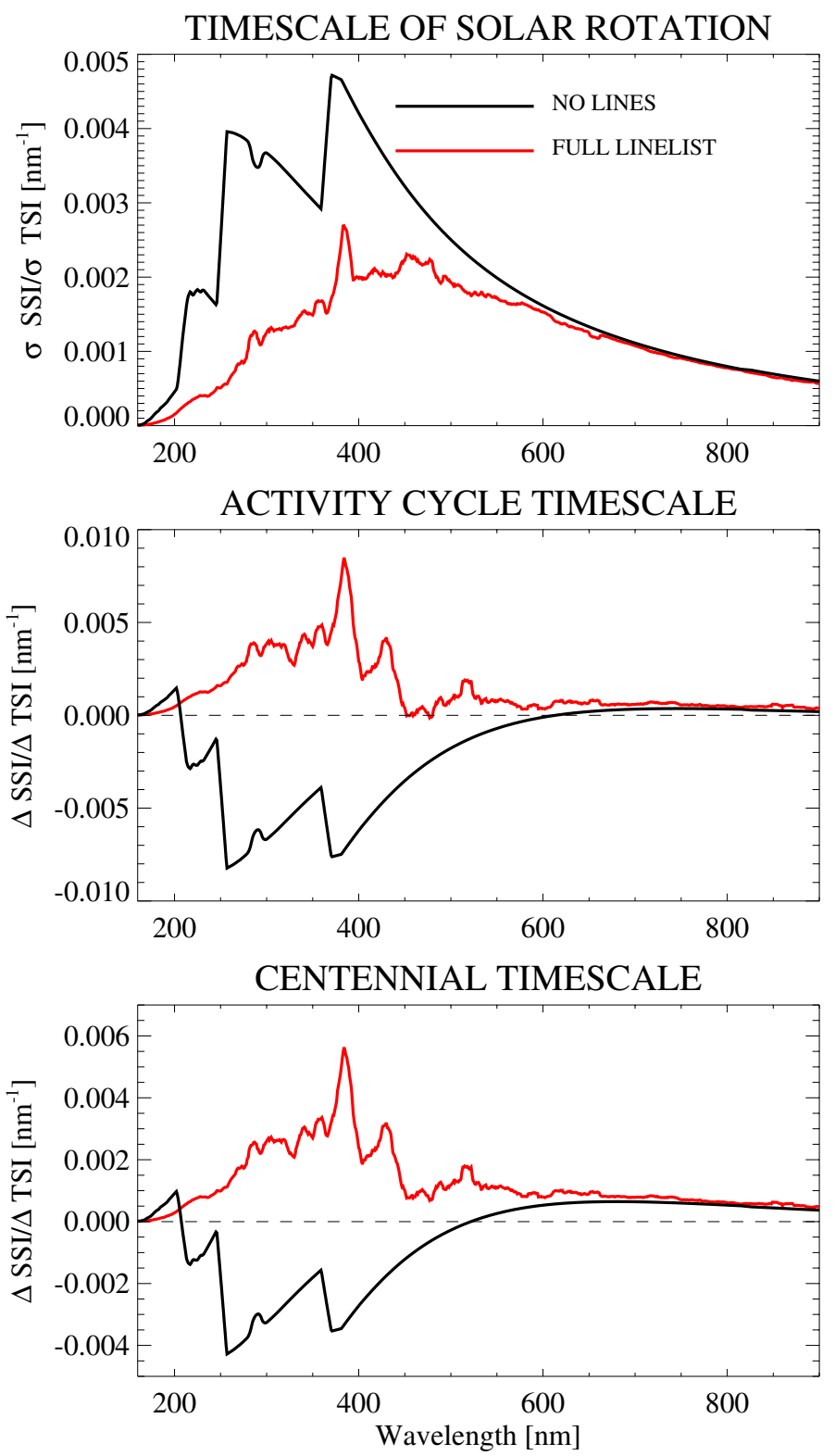

Fig. 4. Same as Fig. 3 but now the black curves represent the values calculated putting the opacity in all Fraunhofer lines to zero.

the assumption of instantaneous chemical equilibrium may fail (Asensio Ramos et al. 2003).

The temperature structures of 1D solar atmospheric models are specifically adjusted to satisfy as many observational constrains as possible. This makes 1D models quite successful at reproducing the observations relevant for calculations of the SSI variability (see e.g. Unruh et al. 1999; Fontenla et al. 2011, and references therein). Additionally, despite their huge potential, self-consistent 3D radiative transfer calculations of the entire solar spectrum based on the results of MHD simulations and capable of reproducing high-resolution observations are not yet readily available (see e.g. Afram et al. 2011). Hence, despite the obvious limitations, the semi-empirical 1D calculations still remain at the forefront of irradiance modelling. At the same time 3D MHD simulations have been gradually reaching a new level of realism and there are also intermediate approaches in between 1D and 3D radiative transfer (Ayres et al. 2006; Holzreuter \& Solanki 2012, 2013). It would be interesting to repeat the exercise presented in this paper when such calculations are applied to modelling of the solar irradiance variability. A study along these lines was recently performed by Criscuoli \& Uitenbroek (2014), who, however, only calculated the facular brightness at continuum wavelengths.

Our result indicates that proper calculations of opacity in the solar atmosphere are of high importance for modelling of the solar irradiance variability. The inclusion of Fraunhofer lines is absolutely crucial for reproducing variations in TSI and SSI at all timescales from the solar rotation period to centuries. For quantitatively accurate computations molecular lines also need to be taken into account, in particular $\mathrm{CN}$ and $\mathrm{CH}$.

Acknowledgements. We thank Greg Kopp for careful reading and constructive and useful comments on the manuscript. The research leading to this paper has received funding from the People Programme (Marie Curie Actions) of the European Union's Seventh Framework Programme (FP7/2007-2013) under REA grant agreement No. 624817. It also got financial support from the BK21 plus program through the National Research Foundation (NRF) funded by the Ministry of Education of Korea. R.V.T. acknowledges support through SNF grant 200020_153301.

\section{References}

Afram, N., Unruh, Y. C., Solanki, S. K., et al. 2011, A\&A, 526, A120 Asensio Ramos, A., Trujillo Bueno, J., Carlsson, M., \& Cernicharo, J. 2003, ApJ, 588, L61

Ayres, T. R. 1989, Sol. Phys., 124, 15

Ayres, T. R., Plymate, C., \& Keller, C. U. 2006, ApJS, 165, 618

Baglin, A., Auvergne, M., Boisnard, L., et al. 2006, in COSPAR Meeting, 36th COSPAR Scientific Assembly, 36, 3749

Ball, W. T., Unruh, Y. C., Krivova, N. A., Solanki, S., \& Harder, J. W. 2011, A\&A, 530, A71

Ball, W. T., Unruh, Y. C., Krivova, N. A., et al. 2012, A\&A, 541, A27

Ball, W. T., Krivova, N. A., Unruh, Y. C., Haigh, J. D., \& Solanki, S. K. 2014, J. Atmos. Sci., 71, 4086

Basri, G., Walkowicz, L. M., \& Reiners, A. 2013, ApJ, 769, 37

Berdyugina, S. V., Solanki, S. K., \& Frutiger, C. 2003, A\&A, 412, 513

Berdyugina, S. V., Braun, P. A., Fluri, D. M., \& Solanki, S. K. 2005, A\&A, 444, 947

Bolduc, C., Charbonneau, P., Barnabé, R., \& Bourqui, M. S. 2014, Sol. Phys., 289, 2891

Bolsée, D., Pereira, N., Decuyper, W., et al. 2014, Sol. Phys., 289, 2433

Bond, G., Kromer, B., Beer, J., et al. 2001, Science, 294, 2130

Bordé, P., Rouan, D., \& Léger, A. 2003, A\&A, 405, 1137

Borucki, W. J., Koch, D., Basri, G., et al. 2010, Science, 327, 977

Castelli, F., \& Kurucz, R. L. 1994, A\&A, 281, 817

Collet, R., Asplund, M., \& Thévenin, F. 2005, A\&A, 442, 643

Criscuoli, S., \& Uitenbroek, H. 2014, ApJ, 788, 151

de Wijn, A. G., Stenflo, J. O., Solanki, S. K., \& Tsuneta, S. 2009, Space Sci. Rev., 144, 275

Deland, M. T., \& Cebula, R. P. 2012, J. Atmos. Sol.-Terr. Phys., 77, 225

Domingo, V., Ermolli, I., Fox, P., et al. 2009, Space Sci. Rev., 145, 337

Ermolli, I., Matthes, K., Dudok de Wit, T., et al. 2013, Atmos. Chem. Phys., 13, 3945

Fabbian, D., Moreno-Insertis, F., Khomenko, E., \& Nordlund, Å. 2012, A\&A, 548, A35

Fligge, M., Solanki, S. K., \& Unruh, Y. C. 2000, A\&A, 353, 380

Floyd, L. E., Cook, J. W., Herring, L. C., \& Crane, P. C. 2003, Adv. Space Res., 31,2111

Fontenla, J., White, O. R., Fox, P. A., Avrett, E. H., \& Kurucz, R. L. 1999, ApJ, 518,480

Fontenla, J. M., Avrett, E., Thuillier, G., \& Harder, J. 2006, ApJ, 639, 441

Fontenla, J. M., Harder, J., Livingston, W., Snow, M., \& Woods, T. 2011, J. Geophys. Res. A, 116, 20108

Fröhlich, C. 2005, Mem. Soc. Astron. It., 76, 731

Fröhlich, C. 2006, Space Sci. Rev., 125, 53

Fröhlich, C. 2009, A\&A, 501, L27

Fröhlich, C. 2013, Space Sci. Rev., 176, 237

Galsgaard, K., \& Nordlund, A. 1996, J. Geophys. Res., 101, 13445

Gray, D. F. 1992, The observation and analysis of stellar photospheres, 2nd edn. (Cambridge University Press)

Gray, L. J., Beer, J., Geller, M., et al. 2010, Rev. Geophys., 48, 4001

Haberreiter, M., Rozanov, E., Rüedi, I., \& Schmutz, W. 2003, in Stellar Atmosphere Modeling, eds. I. Hubeny, D. Mihalas, \& K. Werner, ASP Conf. Ser., 288, 165 
Haigh, J. D. 1994, Nature, 370, 544

Haigh, J. D. 2007, Liv. Rev. Sol. Phys., 4, 2

Hall, J. C., Henry, G. W., Lockwood, G. W., Skiff, B. A., \& Saar, S. H. 2009, AJ, 138,312

Harder, J. W., Fontenla, J. M., Pilewskie, P., Richard, E. C., \& Woods, T. N. 2009, Geophys. Res. Lett., 36, 7801

Holweger, H., Steenbock, W., \& Livingston, W. 1983, Nature, 302, 125

Holzreuter, R., \& Solanki, S. K. 2012, A\&A, 547, A46

Holzreuter, R., \& Solanki, S. K. 2013, A\&A, 558, A20

Hoyt, D. V., Kyle, H. L., Hickey, J. R., \& Maschhoff, R. H. 1992, J. Geophys. Res., 97, 51

Judge, P. G., Lockwood, G. W., Radick, R. R., et al. 2012, A\&A, 544, A88

Knowles, P. J., Werner, H.-J., Hay, P. J., \& Cartwright, D. C. 1988, J. Chem. Phys., 89, 7334

Kodera, K., \& Kuroda, Y. 2002, J. Geophys. Res. A, 107, 4749

Koesterke, L., Allende Prieto, C., \& Lambert, D. L. 2008, ApJ, 680, 764

Kopp, G. 2014, J. Space Weather Space Clim., 4, A14

Kopp, G., Fehlmann, A., Finsterle, W., et al. 2012, Metrologia, 49, 29

Krivova, N. A., Solanki, S. K., Fligge, M., \& Unruh, Y. C. 2003, A\&A, 399, L1

Krivova, N. A., Solanki, S. K., \& Floyd, L. 2006, A\&A, 452, 631

Krivova, N. A., Vieira, L. E. A., \& Solanki, S. K. 2010, J. Geophys. Res., 115,12112

Krivova, N. A., Solanki, S. K., \& Schmutz, W. 2011a, A\&A, 529, A81

Krivova, N. A., Solanki, S. K., \& Unruh, Y. C. 2011b, J. Atmos. Sol.-Terr. Phys., 73, 223

Krupp, B. M. 1974, ApJ, 189, 389

Kuhn, J. R., \& Stein, R. F. 1996, ApJ, 463, L117

Kuhn, J. R., Bush, R. I., Scherrer, P., \& Scheick, X. 1998, Nature, 392, 155

Kupka, F., Piskunov, N., Ryabchikova, T. A., Stempels, H. C., \& Weiss, W. W. 1999, A\&AS, 138, 119

Kupka, F. G., Ryabchikova, T. A., Piskunov, N. E., Stempels, H. C., \& Weiss, W. W. 2000, Balt. Astron., 9, 590

Kurucz, R. L. 1992, Rev. Mex. Astron. Astrofis., 23, 181

Kurucz, R. L. 1993, CD-ROM 13 (Cambridge, Mass.: Smithsonian Astrophysical Observatory)

Lean, J., Rottman, G., Harder, J., \& Kopp, G. 2005, Sol. Phys., 230, 27

Livingston, W., \& Holweger, H. 1982, ApJ, 252, 375

Livingston, W., Wallace, L., White, O. R., \& Giampapa, M. S. 2007, ApJ, 657, 1137

Lockwood, G. W., Skiff, B. A., Henry, G. W., et al. 2007, ApJS, 171, 260

McQuillan, A., Aigrain, S., \& Roberts, S. 2012, A\&A, 539, A137

Mihalas, D. 1978, Stellar Atmospheres, 2nd edn. (San Francisco: Freeman and Company)

Mitchell, Jr., W. E., \& Livingston, W. C. 1991, ApJ, 372, 336
Penza, V., Pietropaolo, E., \& Livingston, W. 2006, A\&A, 454, 349

Radick, R. R., Lockwood, G. W., Skiff, B. A., \& Baliunas, S. L. 1998, ApJS, 118,239

Rauer, H., Catala, C., Aerts, C., et al. 2014, Exp. Astron., 38, 249

Riethmüller, T. L., Solanki, S. K., Berdyugina, S. V., et al. 2014, A\&A, 568, A13

Rutten, R. J., \& Uitenbroek, H. 2012, A\&A, 540, A86

Sánchez Almeida, J., Asensio Ramos, A., Trujillo Bueno, J., \& Cernicharo, J. 2001, ApJ, 555, 978

Scherrer, P. H., Bogart, R. S., Bush, R. I., et al. 1995, Sol. Phys., 162, 129

Schmutz, W., Fehlmann, A., Finsterle, W., Kopp, G., \& Thuillier, G. 2013, AIP Conf. Proc., 1531, 624

Schrijver, C. J., Livingston, W. C., Woods, T. N., \& Mewaldt, R. A. 2011, Geophys. Res. Lett., 38, 6701

Schüssler, M., Shelyag, S., Berdyugina, S., Vögler, A., \& Solanki, S. K. 2003, ApJ, 597, L173

Shapiro, A. I., Schmutz, W., Schoell, M., Haberreiter, M., \& Rozanov, E. 2010, A\&A, 517, A48

Shapiro, A. I., Fluri, D. M., Berdyugina, S. V., Bianda, M., \& Ramelli, R. 2011a, A\&A, 529, A139

Shapiro, A. I., Schmutz, W., Rozanov, E., et al. 2011b, A\&A, 529, A67

Shapiro, A. I., Schmutz, W., Dominique, M., \& Shapiro, A. V. 2013, Sol. Phys., 286, 271

Short, C. I., \& Hauschildt, P. H. 2005, ApJ, 618, 926

Solanki, S. K. 1993, Space Sci. Rev., 63, 1

Solanki, S. K., Inhester, B., \& Schüssler, M. 2006, Rep. Progr. Phys., 69, 563

Solanki, S. K., Krivova, N. A., \& Haigh, J. D. 2013, ARA\&A, 51, 311

Steiner, O. 2005, A\&A, 430, 691

Steiner, O., Hauschildt, P. H., \& Bruls, J. 2001, A\&A, 372, L13

Stenflo, J. O. 2013, A\&ARv, 21, 66

Stenflo, J. O., Keller, C. U., \& Gandorfer, A. 1998, A\&A, 329, 319

Thuillier, G., Claudel, J., Djafer, D., et al. 2011, Sol. Phys., 268, 125

Thuillier, G., Bolsée, D., Schmidtke, G., et al. 2014, Sol. Phys., 289, 1931

Thuillier, G., Harder, J., Shapiro, A., et al. 2015, Sol. Phys., 289, 2433

Uitenbroek, H., \& Criscuoli, S. 2011, ApJ, 736, 69

Unruh, Y. C., Solanki, S. K., \& Fligge, M. 1999, A\&A, 345, 635

Vitas, N., Viticchiè, B., Rutten, R. J., \& Vögler, A. 2009, A\&A, 499, 301

Wallace, L., Hinkle, K., Li, G., \& Bernath, P. 1999, ApJ, 524, 454

Willson, R. C., Gulkis, S., Janssen, M., Hudson, H. S., \& Chapman, G. A. 1981, Science, 211,700

Woods, T. N., Tobiska, W. K., Rottman, G. J., \& Worden, J. R. 2000, J. Geophys. Res., 105, 27195

Yeo, K. L., Solanki, S. K., \& Krivova, N. A. 2013, A\&A, 550, A95

Yeo, K. L., Krivova, N. A., \& Solanki, S. K. 2014a, Space Sci. Rev., 186, 137

Yeo, K. L., Krivova, N. A., Solanki, S. K., \& Glassmeier, K. H. 2014b, A\&A, 570, A85 